\title{
Bur open The developmental origins of ageing: study protocol for the Dutch famine birth cohort study on ageing
}

\author{
Susanne R de Rooij, ${ }^{1}$ Tessa J Roseboom ${ }^{1,2}$
}

To cite: de Rooij SR, Roseboom TJ. The developmental origins of ageing: study protocol for the Dutch famine birth cohort study on ageing. BMJ Open 2013;3: $\mathrm{e} 003167$ doi:10.1136/bmjopen-2013003167

- Prepublication history for this paper is available online To view these files please visit the journal online (http://dx.doi.org/10.1136/ bmjopen-2013-003167).

Received 6 May 2013 Accepted 8 May 2013

This final article is available for use under the terms of the Creative Commons Attribution Non-Commercial 2.0 Licence; see http://bmjopen.bmj.com

\section{${ }^{1}$ Department of Clinical} Epidemiology and Biostatistics, Academic Medical Centre, University of Amsterdam, Amsterdam, The Netherlands

2Department of Obstetrics and Gynaecology, Academic Medical Centre, University of Amsterdam, Amsterdam, The Netherlands

Correspondence to Dr Susanne R de Rooij; s.r.derooij@amc.uva.nl

\section{ABSTRACT}

Introduction: Evidence from animal studies suggest that the rate of ageing may be influenced not only by genetic and lifestyle factors, but also by the prenatal environment. We have previously shown that people who were exposed to famine during early gestation performed worse on a selective attention task, which may be a first sign of cognitive decline, and were on average 3 years younger at the time of coronary artery disease diagnosis. Women in this group seem to die at a younger age. We hypothesise that an accelerated ageing process, set in motion by the poor prenatal environment, underlies these findings.

Methods and analysis: The Dutch Famine Birth Cohort consists of 2414 men and women born in Amsterdam as term singletons around the time of the Dutch famine. In a subsample of 150 cohort members, who now are about 68 years of age, we are currently measuring cognitive decline and the incidence of white matter hyperintensities and cerebral microbleeds (through MRI), incidence of fractures, grip strength and physical performance, visual acuity and incidence of cataract operations. In this same subgroup, we will assess telomere length, oxidative stress and inflammatory status as potential underlying mechanisms. Furthermore, in the entire cohort, we will assess mortality as well as hospital admissions for age-related diseases up to the age of 68 years.

Ethics and dissemination: The study was approved by the local medical ethics committee (Academic Medical Centre, University of Amsterdam) and is being carried out in agreement with the Declaration of Helsinki. All participants give written informed consent. Study findings will be widely disseminated to the scientific public as well as to the medical society and general public.

\section{INTRODUCTION}

The number of elderly people is growing rapidly worldwide. Although ageing is an inevitable biological process, there are large variations in the rate at which people age. With so many people getting into old age, understanding the biology of healthy ageing is now more relevant than ever.

\section{ARTICLE SUMMARY}

Article focus

- The present study is aimed at investigating the developmental origins of ageing.

- It is hypothesised that undernutrition during early-gestation programmes accelerated ageing through reduced functional capacity; reduced somatic repair; impaired antioxidant systems and initiation of chronic inflammation.

\section{Key messages}

- The study results will potentially show that men and women who were conceived during the Dutch famine-and had thus been exposed to undernutrition during their earliest development -age more quickly and that the accelerated ageing process is due to the programming of basic biological processes such as cell metabolism, senescence and inflammation.

- Knowledge on the contribution of early nutrition to the ageing process is relevant for the investigation of the ageing process itself and for the prevention and intervention strategies aimed at slowing the ageing process.

Strengths and limitations of this study

- The study uses the unique opportunity provided by the Dutch famine to investigate whether the ageing process in humans is affected by earlynutritional factors and what are the underlying biological processes.

- Exposure to famine during gestation is based on the date of birth and the official daily food rations. Individual data on famine exposure are not available. This could potentially lead to underestimation of the effects of prenatal famine exposure on ageing.

Research in the field of ageing has mainly focused on genetic and lifestyle factors. Several genes have been identified that contribute to the rate of ageing, although the effects are generally small. Lifestyle factors such as diet and smoking are well-established factors adding to the variation seen in ageing. One of the most extensively studied and successful ways to elongate lifespan is 
moderate dietary restriction. ${ }^{1}$ Studies across different species have shown that moderate dietary restriction is associated with a longer lifespan. Surprisingly, however, when diet is restricted in prenatal life, the effect is completely the opposite and reduces the lifespan considerably. Mice that were undernourished prenatally had a $25 \%$ shorter lifespan than those who had not been undernourished before birth. As such, the effects of dietary restriction in prenatal life are larger than those of postnatal diet. ${ }^{2}$ These findings suggest that the early environment may have lasting effects on the ageing process.

Finch $^{3}$ has proposed four mechanisms by which adversity during development may accelerate ageing: (1) Reduced functional capacity; (2) reduced capacity for somatic repair and regeneration; (3) impaired antioxidant systems and (4) initiation of chronic inflammation. Experiments in animals have shown that prenatal undernutrition indeed altered the expression of genes involved in the ageing process; it reduced the telomere length, impaired the antioxidant defences ${ }^{4}$ and increased the rates of basal inflammation. ${ }^{5}$

Although evidence for a role of early nutritional factors in ageing has been shown in a variety of organisms, there is no direct evidence in humans that early nutritional factors affect the rate at which people age. ${ }^{4} 6$ There is some indirect evidence though. Observational studies in humans have shown that small size at birthas a measure of suboptimal conditions in utero-is associated with markers of ageing in a number of different body systems, including the brain, eye, ear, muscle and bone. ${ }^{7-9}$ It has been suggested that these associations reflect nutritional programming, but direct evidence is lacking. Direct evidence from human studies is imperative to establish a role for early nutrition in the ageing process and to unravel the biological processes by which early nutrition may programme the rate of ageing of the human body. Obviously, experimentally modulating early nutrition in humans is difficult and such studies are thus difficult to come by.

The Dutch Famine Birth Cohort Study is an 'experiment of history' which provides a unique opportunity to investigate the effects of prenatal undernutrition on the ageing process. During the winter of 1944-1945, the western part of the Netherlands was struck by a period of severe food scarcity. The previously and subsequently well-nourished Dutch population's daily rations dropped acutely to as little as 400-800 calories during the 5 months of famine. The famine was a humanitarian disaster, but it left an opportunity to study the effects of maternal malnutrition on the offspring's health and ageing in later life. Using birth records of babies born around the time of the Dutch famine in the Wilhelmina Gasthuis in Amsterdam, we have been able to investigate the long-term consequences of prenatal undernutrition. We have shown that those who were conceived during the famine-and had thus been undernourished during the earliest stages of their development-have an increased risk for coronary heart disease, diabetes, an atherosclerotic lipid profile, altered clotting and breast cancer (table 1$).{ }^{10}$

What stands out here is that all these diseases are age-associated diseases. This could be due to the altered structural development of body organs and systems, rendering the person more vulnerable to these diseases. Alternatively, or additionally, prenatal exposure to famine may alter basic biological processes, such as cell metabolism, cell senescence and inflammation processes, thereby setting in motion an accelerated ageing process.

We have found some suggestions that those who were conceived during the famine-and had thus been undernourished during the earliest stages of their development-age more quickly than those who had not been undernourished in utero. Men and women who were conceived during the famine had doubled rates of coronary heart disease, which occurred on average 3 years earlier than among those who had not been undernourished prenatally. ${ }^{11}$ Cognition may also deteriorate faster. People who had been exposed to famine during early gestation performed worse on a selective attention task. ${ }^{12}$ Interestingly, selective attention is a cognitive ability that is one of the first to decline with age and has been shown to be a strong predictor for conversion to Alzheimer's' disease even before memory deficits

Table 1 Prevalence of age-associated diseases according to prenatal famine exposure

\begin{tabular}{|c|c|c|c|c|c|c|c|}
\hline & \multirow[b]{2}{*}{$\begin{array}{l}\text { Born } \\
\text { before }\end{array}$} & \multicolumn{6}{|c|}{ Exposure to famine } \\
\hline & & $\begin{array}{l}\text { In late } \\
\text { gestation }\end{array}$ & $\begin{array}{l}\text { In } \\
\text { mid-gestation }\end{array}$ & $\begin{array}{l}\text { In early } \\
\text { gestation }\end{array}$ & $\begin{array}{l}\text { Conceived } \\
\text { after }\end{array}$ & All & $\mathbf{N}$ \\
\hline CVD (\%) & 3.8 & 2.5 & 0.9 & $8.8^{*}$ & 2.6 & 3.3 & 736 \\
\hline Type 2 diabetes (\%) & 15.1 & 14.2 & 13.8 & 18.9 & 12.6 & 14.4 & 783 \\
\hline LDL/HDL & 2.9 & 2.8 & 2.7 & $3.3^{*}$ & 2.9 & 2.9 & 704 \\
\hline $\begin{array}{l}\text { Breast cancer in women } \\
(\%)\end{array}$ & 2.8 & 3.7 & 3.9 & $8.7^{\star}$ & 0.8 & 3.2 & 475 \\
\hline Depression in men (\%) & 5.1 & 8.2 & 6.7 & 13.3 & 6.1 & 6.8 & 367 \\
\hline Cognition (score) & 42.3 & 36.5 & 40.0 & $27.5^{\star}$ & 43.9 & 42.3 & 714 \\
\hline
\end{tabular}


were present. ${ }^{13}$ Furthermore, preliminary evidence suggests that women exposed to famine during early gestation seem to die younger. ${ }^{14}$

With this study, we propose to use the unique opportunity provided by the Dutch famine to solve an unresolved question in the field of ageing research: is the ageing process in humans affected by early environmental factors and what are the underlying biological processes? We aim to investigate whether undernutrition in early gestation indeed affects the rate of ageing at various levels and whether this is set in motion by the programming of basic cellular processes.

We hypothesise that undernutrition during earlygestation programmes accelerated ageing through reduced functional capacity; reduced somatic repair; impaired antioxidant systems and initiation of chronic inflammation. Men and women who were conceived during the Dutch famine-and had thus been exposed to undernutrition during their earliest developmentaged more quickly. The accelerated ageing process is manifested at different levels:

1. The whole body level, as shown by increased mortality and increased hospital admissions for age-related disease.

2. The organ/system level, as shown by increased levels of ageing markers in the brain, bone, muscle and eye.

3. The cell level, as shown by increased oxidative stress, decreased the telomere length and increased chronic inflammation.

4. The gene expression level, as shown by altered levels of several genes associated with ageing.

\section{METHODS AND ANALYSIS}

\section{Dutch Famine Birth Cohort}

The Dutch Famine Birth Cohort is a historical cohort of originally 2414 men and women who were born between 1 November 1943 and 28 February 1947 in the Wilhelmina Gasthuis, a local hospital in Amsterdam. ${ }^{15}$ People were included in the cohort if they were born alive as a singleton after a pregnancy duration of at least 259 days and if a medical birth record could be retrieved. Preterm babies were thus excluded. Between 1994 and 1996, the population registry traced 2155 $(89 \%)$ of the 2414 eligible persons. Of these, 265 had died and 199 had emigrated from the Netherlands. The population registry asked permission of eligible persons to give their address to the researchers. A total of 164 persons refused, resulting in a cohort of 1527 at the start of the study in 1995. Since then, three rounds of data collection have been performed, in 1995-1997, in 20022004 and in 2008-2009.

\section{Exposure to famine}

Prenatal exposure to undernutrition is defined as a daily maternal intake of less than 1000 calories during at least 13 weeks (about one-third of the gestational period).
The official daily food rations for the general population older than 21 years in Amsterdam (available from the national bureau for food distribution at that time) are used to estimate maternal food intake. ${ }^{16}$ In addition to the official rations, food also came from other sources (eg, church organisations, central kitchens and the 'black market'). People may have had access up to double the rationed amount at the peak of the famine. The rations do, however, adequately reflect the fluctuation of food availability during the famine. ${ }^{17}$ Children younger than 1 year were relatively protected, as their rations never fell below 1000 calories. Before the famine, pregnant women received extra rations, but during the famine these extra supplies were no longer available.

On the basis of this, babies born between 7 January 1945 and 8 December 1945 are considered to have been exposed to undernutrition in utero. We previously delineated periods of 16 weeks each to differentiate between those exposed in late gestation (born between 7 January and 28 April 1945), in mid gestation (born between 29 April and 18 August 1945) and in early gestation (born between 19 August and 8 December 1945). In the proposed study, we will focus on babies who have been exposed to famine during early gestation, since we have found most effects on health (including mortality, coronary heart disease, breast cancer, depression and cognition) among this group. We will compare this group of people with two groups of unexposed people: people born before 7 January 1945 (only exposed to famine during the first year of life and relatively protected during this period) and people born after 8 December 1945 (conceived after the famine and thus never experienced famine).

\section{Study population}

Mortality and hospital admission for age-related diseases will be studied in the entire eligible cohort of 2414 individuals, by linking to the national mortality and hospital admission registries. All other measurements are performed in a subsample of 150 cohort members ( 50 born before the famine, 50 exposed to the famine in early gestation and 50 conceived and born after the famine; $\mathrm{n}=50$ is based on power calculations, see below). The subsample is randomly drawn from the group of cohort members who participated in the 2002-2004 clinical study $(n=740)$. This will enable us to investigate rates of cognitive decline as well as age-related increases in blood pressure and other age-related changes in cardiometabolic markers.

\section{Measurements}

For an overview of our study hypothesis and planned measurements, (see figure 1).

At whole body level

Mortality: Accelerated ageing ultimately leads to a shorter lifespan. Animal experiments have shown that 
Figure 1 Overview of study hypothesis and measurements.

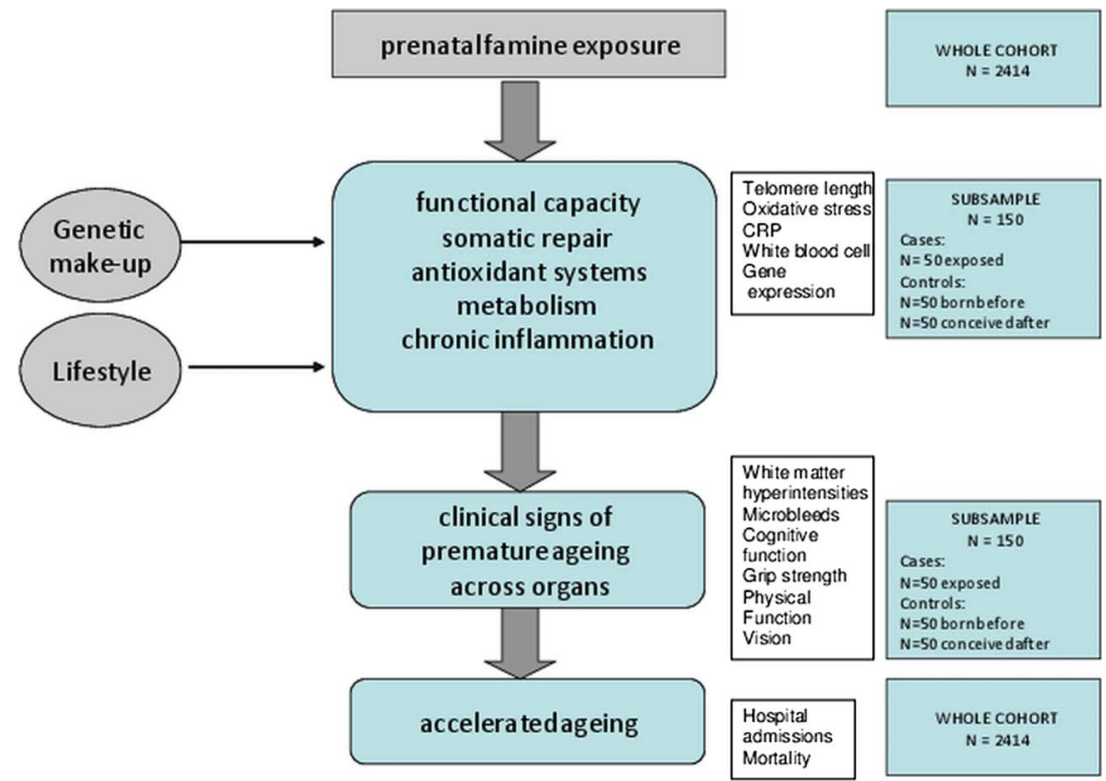

diet restriction during pregnancy and shortly after birth shortens the lifespan in the offspring. ${ }^{2}{ }^{18}$ Small size at birth and at 1 year of age, as indirect measures of a poor start in life, have also been associated with a decreased lifespan. ${ }^{19}$ We have previously investigated mortality in the Dutch Famine Birth Cohort up to the ages of 50 and 57, but could not demonstrate an effect of prenatal famine exposure on mortality. ${ }^{20}{ }^{21}$ However, this was possibly due to the relatively young age of the cohort at these times, because recent data suggest that, up to the age of 62 years, women exposed to famine in early gestation had a higher overall adult, cardiovascular, cancer and breast cancer mortality risk. ${ }^{14}$ In the current proposed study, we aim to study all-cause mortality and cause-specific mortality up to the age of 68 for the whole cohort. Mortality data and causes of death for these people will be retrieved by linking the cohort to Statistics Netherlands.

Age-related diseases: In previous Dutch famine studies, it has been shown that famine exposure during early gestation is associated with increased risk for coronary heart disease, type 2 diabetes, an atherosclerotic lipid profile, altered clotting and breast cancer, all age-associated diseases. ${ }^{10}$ In the present study, we will assess the presence of other clearly age-associated diseases, including stroke, fractures and cataract. Stroke is a highly prevalent age-related brain disease and a common cause for disability and invalidism in the elderly. Two studies investigating low-birth weight as a proxy for a poor-prenatal environment have shown adverse associations between low-birth weight and the incidence of stroke at older age. $^{22} 23$ Ageing is associated with bone loss and osteoporosis, a disease that leads to an increased risk of fracture particularly affecting older people. The Helsinki Birth Cohort Study has shown that shortness at birth was associated with increased hip fracture risk. ${ }^{24}$ Visual acuity generally declines with age and several eye diseases, such as cataract, may emerge. Cataract is a cloudy area in a part or the whole of the lens. There is some indirect evidence that a poor start early in life leads to increased symptoms of ageing in the eye. Lower weight at 1 year of age is an indicator of poor early growth. In men and women who were born in Hertfordshire (UK), lower weight at 1 year of age was associated with an increased incidence of cataract at the age of 64-74 years. ${ }^{8}$ We will study the incidence of stroke, fractures and cataract operations in the entire cohort by linkage to hospital discharge diagnoses requested from the National Medical Registry.

\section{Measurements at organ level}

All the measurements described below will be performed in the subsample of 150 participants.

Brain: One of the major brain-associated ageing symptoms is that of cognitive decline. Evidence from animal experiments shows that adverse early-life circumstances affect cognitive and behavioural function in older age. Rats that were undernourished during the perinatal period showed slowness in learning a maze, behavioural rigidity and high emotionality resembling the characteristics of rats at old age. ${ }^{25}$ Associated with cognitive decline in older age are white matter hyperintensities and cerebral microbleeds in the brain. A very small human study by Hulshoff-Pol et $a l^{26}$ suggested an increased incidence of white matter hyperintensities after prenatal famine exposure. They took 18 schizophrenic patients of whom half had been exposed to the Dutch famine during early gestation and matched them to 18 non-schizophrenic people of whom again half had been exposed to famine during early gestation. Five of the 18 famine-exposed participants showed white matter hyperintensities compared with one in the unexposed 
group. This finding is highly interesting and requires further investigation in a larger sample. We will measure cognitive decline and function. To measure cognitive decline over a period of about 10 years, we will repeat the cognitive tasks we performed between 2002 and 2004. At that time, we assessed a number of cognitive abilities. Two cognitive tasks, a Stroop-like colour-word conflict task and a mirror drawing task, were performed as part of a psychological stress protocol. Two other tasks, a story repeating task and the Alice Heim 4 test, were applied to measure memory and general intelligence. Data suggested that performance on the Stroop task measuring selective attention was worse in those exposed to famine during early gestation. ${ }^{12}$ We will run these tests again and measure the difference in performance. Additional tests that will be included are: Trail making A and B (measuring processing speed, attention and mental flexibility) and the 15 Words test (measuring memory). Incidence of white matter hyperintensities and cerebral microbleeds will be examined with standardised MRI of the brain. The total number and volume of white matter signal hyperintensities and total number of microbleeds will be assessed as well as the total cortical volume, hippocampal volume and lacunar volume.

Bone: As reported above, small size at birth was associated with hip fracture risk. ${ }^{24}$ Frailty may thus have its origins before birth. A questionnaire will be used to determine whether the participant has ever been diagnosed with osteoporosis, whether there is a history of fracture(s) and, if so, at what age the fractures occurred. In addition, we will study the incidence of fractures in the entire cohort by using hospital discharge diagnoses linked to the National Medical Registry.

Muscle: Sarcopenia, the loss of skeletal muscle mass and strength with age, has serious consequences for the quality of life in older people. Several cohort studies have shown that grip strength at a later age is reduced in those who were born small at birth or at 1 year of age. Cohorts included older men and women born in Hertfordshire, UK, and in Finland and middle-aged British men and women. ${ }^{8} 2728$ These results suggest that sarcopenia may have its origins before birth. Further evidence for this notion comes from a study in which it was shown that not only grip strength but also muscle mass was decreased in the elderly with a low-birth weight. ${ }^{29}$ Physical performance declines with age and loss of muscle mass and strength. A study performed in the UK in older men who were small at birth or at 1 year of age showed an association with poorer standing balance. ${ }^{30}$ This suggests that a decline in physical performance may be another aspect of the ageing process affected by poor early-life circumstances. As an indicator of muscle strength, grip strength of the dominant hand (maximum of three measurements) will be measured by means of a Jamar handgrip dynamometer. We will also measure physical performance with a number of tests, including a 3 min walk, chair rises and standing balance, as well as with a physical performance questionnaire.
Eye: As has already been referred to above, visual acuity generally declines with age. Besides studying the incidence of cataract operations in the entire cohort, we will measure visual acuity with standard Snellen charts in the subsample.

\section{Measurements at cell level}

All the measurements described below will be performed in the subsample of 150 participants.

Telomere length: Telomeres are DNA repeat sequences that cap the ends of chromosomes. Telomeres decrease in length by a predictable amount with each cell division. Recently, it has also been shown that telomere length declines in response to oxidative damage (see below). When telomere length becomes critically short, the cell is no longer able to replicate and enters cellular senescence. Telomere length has been postulated as either a mechanism of or a marker for cellular ageing and longevity regulation. It has been associated with many diseases related to ageing as well as to the lifespan itself. ${ }^{31}$ Experiments in rats showed that feeding rat mothers a protein-restricted diet leads to reduced telomere length in the kidney, aortic tissue and pancreatic islet tissue in the offspring, possibly explaining the shortened lifespan in these animals. ${ }^{32-34}$ We will investigate telomere length in DNA extracted from isolated white blood cells.

Oxidative stress: Oxidative stress occurs when there is an imbalance between the production of reactive oxygen species (ROS), a natural by-product of the normal metabolism of oxygen in the body, and the ability of the body to neutralise ROS. Oxidative stress can result in oxidative damage to the cells, tissues or organs and can also cause telomere shortening independently of cell division. Oxidative stress has been implicated in ageing and many age-associated diseases, such as cancer, diabetes, cardiovascular disease and neurodegenerative diseases. It has also been suggested as one of the causes of cellular ageing. ${ }^{35}$ Studies in the progeny of rats who received a protein-restricted diet in utero showed an increased production of ROS and a decreased oxidative defence resulting in increased oxidative stress. ${ }^{33} 34$ Prepubertal children who were born either small or large for gestational age were shown to have increased oxidative stress quantified by impaired oxidant/antioxidant status. ${ }^{36}$ We will measure oxidative stress quantified by two oxidative stress markers. As a first marker, urinary F2-IsoP, a biomarker for systemic, whole body oxidative stress over time, will be assessed. As a second marker, a single cell gel electrophoresis assay (comet assay) will be applied. This assay measures DNA damage at the level of a single cell. We will use white blood cells for this.

Chronic basal inflammation: There is increasing evidence that chronic upregulation of pro-inflammatory mediators are implicated in the ageing process and diverse age-related diseases including cardiovascular disease, diabetes and dementia. ${ }^{37}$ Experimental evidence suggests that this chronic upregulation may be 
programmed by undernutrition in utero. Desai et at showed that a $50 \%$ food restriction of the maternal diet led to increased basal inflammation but decreased inflammation responses to inflammatory provoking stimuli in the offspring. Several indices of reduced fetal growth have been associated with increased levels of basal $\mathrm{C}$ reactive protein (CRP), a major inflammatory factor, in adulthood. ${ }^{38} 39$ For example, a large population-based study showed that a $1 \mathrm{~kg}$ decrease in birthweight was associated with an $11 \%$ increase in CRP in adults aged $30-59$ years. ${ }^{39}$ The primary cell effectors of inflammation, leucocytes, have also been shown to be associated with small size at birth. ${ }^{40}$ In a study of over 5000 people, total leukocyte count at the age of 31 years was higher in those with lower birth weights. We will measure basal inflammation status by assessment of two important indicators of inflammation status: CRP and total white cell count.

\section{Measurements at gene expression level}

We aim to investigate gene expression levels of several genes associated with ageing. As this part of the study is planned to be performed at the end of the entire study, we will let the choice of genes to be assessed depend on the discovery of novel genes that will be implicated in the ageing process. Currently, SIRT1 and p16, both implicated in accelerated ageing in mice after protein restriction during fetal life and subsequent overfeeding in postnatal life, are strong candidates. ${ }^{34} 41$

\section{Potential covariates/confounders/mediators}

Height will be measured with a fixed stadiometer and weight with portable Tefal scales. Waist and hip circumferences will be measured with a flexible tape measure. Blood will be drawn for the assessment of the lipid profile (low-density lipoprotein-cholesterol, high-density lipoprotein-cholesterol, triglycerides) and the glucose and insulin levels. Participants will be interviewed and asked about the socioeconomic status (SES) indicators, medical history, lifestyle (smoking, alcohol consumption, diet, exercise) and use of medication. Standardised questionnaires will be used. We will administer the Hospital Anxiety and Depression Scale to assess the presence of depression symptoms.

\section{Statistical methods and power analyses}

We will start all analyses by checking whether the two control groups, those born before the famine and those conceived after the famine, do not differ on the outcomes investigated. Subsequently, we will use linear and logistic regression analyses to investigate associations between prenatal exposure to famine and incidence of white matter hyperintensities and cerebral microbleeds, cognitive test outcomes, history of fracture, grip strength and physical performance, vision-outcomes, telomere length, oxidative stress and inflammation markers. Cox proportional hazards models will be used to explore the influence of prenatal famine exposure on all-cause and cause-specific cumulative adult mortality. As there are known differences in ageing between men and women as well as different effects of fetal programming in men and women, we will look for sex differences in all analyses. Furthermore, analyses will be adjusted for suitable potential covariates and confounding variables. Data with skewed distributions will be transformed to attain normality where possible.

On the basis of a number of 50 participants in the group of people exposed to famine during early gestation (cases) and 100 controls, we expect to have sufficient power to find meaningful differences in all the outcomes. We give a number of examples. With regard to the MRI measurements, we will have $80 \%$ power with a significance level of $5 \%$ to find about a half SD difference in the total number of white matter hyperintensities between the group of participants exposed to famine in early gestation and those unexposed during gestation. A half SD difference was found to correspond to the difference between groups of elderly people without any cognitive impairment and with mild cognitive impairment, based on an extensive cognitive test battery. ${ }^{42}$

On the basis of a significance level of $5 \%$, we have $80 \%$ power to detect differences between those exposed to famine during early gestation and those unexposed during gestation from $13 \%$ up in telomere length (based on an SD of $0.24 \mathrm{~T} / \mathrm{S}$ units). For comparison, in a follow-up study, it was shown that women with about a $30 \%$ shorter telomere length at baseline were 2.3 times more likely to die from CVD over the next 12 years. ${ }^{43}$

Again, based on a significance level of $5 \%$, we have $80 \%$ power to detect differences between those exposed to famine during early gestation and those unexposed during gestation from $12 \%$ up in the oxidative stress marker comet tail moment (based on an SD of 0.31). This percentage roughly corresponds to the difference in this oxidative stress marker between those who are of normal weight and those who are obese. ${ }^{44}$

Furthermore, based on the total cohort, we have $80 \%$ power to find an HR of 1.35 for all-cause mortality.

\section{Study procedure and planning}

Subsample participants are invited by letter and, upon agreement, are visited at home where measurements of blood pressure, anthropometrics, grip strength, physical performance, vision and cognitive ability are being performed. Participants are also being interviewed about SES, lifestyle and medical history. Subsequently, they are invited to visit the clinic for blood withdrawal, urine collection and MRI scanning. Travelling costs are reimbursed and participants are offered a small token of appreciation for participation in the study. Data collection has been ongoing since November 2012 and is scheduled to be finished within 1 year. Data analyses and manuscript drafting will be performed within the period from the beginning of 2014 onwards. 


\section{ETHICS AND DISSEMINATION}

\section{Ethics and safety considerations}

The study is being conducted according to the principles of the Declaration of Helsinki (version October 2008) and in accordance with the Medical Research Involving Human Subjects Act (WMO). The study was approved by the local medical ethics committee (Academic Medical Centre, University of Amsterdam). All participants give written informed consent. The study imposes no risks on the participants. Taking a venous blood sample $(25 \mathrm{~mL})$ is a small discomfort, but safe. A small percentage of participants may experience claustrophobia or anxiety during the MRI scan. It will therefore be stressed to participants that the MRI scan can be stopped at any moment. The total testing time will be about $6 \mathrm{~h}(4 \mathrm{~h}$ at home and $2 \mathrm{~h}$ in the clinic, including breaks).

\section{Dissemination}

Study results will be disseminated to the scientific public by presentation at national and international conferences and by publication in peer-reviewed journals. The concept that, during critical periods of fetal development, environmental factors such as undernutrition may cause permanent adaptations in the structure and function of the fetus, thereby increasing risk for disease in later life, has been recognised in the fields of developmental biology and medicine. It has become known as developmental programming or the fetal origins hypothesis. ${ }^{45}{ }^{46}$ Evidence for this hypothesis has increased over the last decades. The concept has been sparsely implicated in the field of ageing research though. The present study proposal therefore constitutes a valuable interdisciplinary contribution to the field of gerontology. We therefore aim to present the study findings in conferences dedicated to both fields of research and publish papers in broadly oriented scientific journals.

The study findings will also be relevant from a societal perspective. Study results may show that prenatal undernutrition is associated with accelerated ageing and longevity. This would confirm previous evidence from our studies in the Dutch Famine Birth Cohort that nutrition during pregnancy is important for health in later life. That it is not only important in the prevention of several diseases, but also accelerates the ageing process, adds even more relevance to the notion that pregnant women should consume healthy diets. This knowledge could be used for public-health strategies. It is a powerful message that we can influence the ageing process at the start of life. Also, it may well be more feasible to change the diet of women during pregnancy, which is only a short period of time and for the lifelong good of their child, than to change the lifelong diet and lifestyle of adults. Therefore, we aim to also disseminate the study findings among nutritionists, midwives and gynaecologists who are in a position to advise (future) pregnant women about nutrition and the public in general by publishing results in national newspapers and (pregnant) women magazines.

\section{CONCLUSION}

In conclusion, the results of the present ongoing study will provide new knowledge on the contribution of early nutrition to the ageing process. It will show whether the rate of ageing across the system and the organ level is affected by prenatal undernutrition and whether this is due to programming of the basic biological processes such as cell metabolism, senescence and inflammation. This knowledge is relevant for the investigation of the ageing process itself and for the prevention and intervention strategies aimed at slowing the ageing process.

Contributors SRdR and TJR are the chief investigators who designed and established this research study. SRdR and TJR equally contributed to the writing of this manuscript and both have critically reviewed and approved the final version.

Funding The Dutch famine birth cohort study is currently funded by the European Community FP7 HEALTH, Project 279281 (BRAINAGE).

Competing interests None.

Ethics approval Local Medical Ethics Committe.

Provenance and peer review Not commissioned; internally peer reviewed.

Data sharing statement No additional data are available.

\section{REFERENCES}

1. Fontana L, Partridge L, Longo VD. Extending healthy life spanfrom yeast to humans. Science 2010;328:321-6.

2. Ozanne SE, Hales CN. Lifespan: catch-up growth and obesity in male mice. Nature 2004;427:411-12.

3. Finch CE. The biology of human longevity. Oxford: Elsevier, 2008.

4. Barnes SK, Ozanne SE. Pathways linking the early environment to long-term health and lifespan. Prog Biophys Mol Biol 2011;106:323-36.

5. Desai M, Gayle DA, Casillas E, et al. Early undernutrition attenuates the inflammatory response in adult rat offspring. J Matern Fetal Neonatal Med 2009;22:571-5.

6. Brakefield PM, Gems D, Cowen T, et al. What are the effects of maternal and pre-adult environments on ageing in humans, and are there lessons from animal models? Mech Ageing Dev 2005;126:431-8.

7. Shenkin SD, Bastin ME, Macgillivray TJ, et al. Birth parameters are associated with late-life white matter integrity in community-dwelling older people. Stroke 2009;40:1225-8.

8. Aihie Sayer A, Rauf A, Evans JR, et al. Reduced growth before one year of age is associated with more rapid aging. Age Ageing 1996;25:7.

9. Dennison EM, Syddall HE, Sayer AA, et al. Birth weight and weight at 1 year are independent determinants of bone mass in the seventh decade: the Hertfordshire cohort study. Pediatr Res 2005;57:582-6.

10. Roseboom T, de Rooij S, Painter R. The Dutch famine and its long-term consequences for adult health. Early Hum Dev 2006;82:485-91.

11. Painter RC, de Rooij SR, Roseboom TJ, et al. Early onset of coronary artery disease after prenatal exposure to the Dutch famine. AJCN 2006;84:322-7.

12. de Rooij SR, Wouters $\mathrm{H}$, Yonker JE, et al. Prenatal undernutrition and cognitive function in late adulthood. Proc Natl Acad Sci USA 2010;107:16881-6.

13. Balota DA, Tse CS, Hutchison KA, et al. Predicting conversion to dementia of the Alzheimer's type in a healthy control sample: the power of errors in Stroop color naming. Psychol Aging 2010;25:208-18.

14. van Abeelen AF, Veenendaal MV, Painter RC, et al. Survival effects of prenatal famine exposure. Am J Clin Nutr 2012;95:179-83.

15. Ravelli ACJ, van der Meulen JHP, Michels RPJ, et al. Glucose tolerance in adults after prenatal exposure to famine. Lancet 1998;351:173-7.

16. Burger GCE, Sandstead HR, Drummond JC. Malnutrition and starvation in Western Netherlands, September 1944 to July 1945. Part I and II. The Hague: General State Printing Office, 1948.

17. Trienekens G. Tussen ons volk en de honger. 1st edn. Utrecht: Matrijs, 1985. 
18. Aihie Sayer A, Dunn R, Langley-Evans S, et al. Prenatal exposure to a maternal low protein diet shortens life span in rats. Gerontology 2001;47:9-14.

19. Osmond C, Barker DJP, Winter PD, et al. Early growth and death from cardiovascular disease in women. Br J Obstet Gynaecol 1993;307:1519-24.

20. Roseboom TJ, van der Meulen JHP, Osmond C, et al. Adult survival after prenatal exposure to the Dutch famine 1944-45. Paediatr Perinat Epidemiol 2001;15:220-5.

21. Painter RC, Roseboom TJ, Bossuyt PM, et al. Adult mortality at age 57 after prenatal exposure to the Dutch famine. Eur J Epidemiol 2005;20:673-6.

22. Lawlor DA, Ronalds G, Clark $\mathrm{H}$, et al. Birth weight is inversely associated with incident coronary heart disease and stroke among individuals born in the 1950s: findings from the Aberdeen Children of the 1950s prospective cohort study. Circulation 2005;112:1414-18.

23. Rich-Edwards JW, Kleinman K, Michels KB, et al. Longitudinal study of birth weight and adult body mass index in predicting risk of coronary heart disease and stroke in women. BMJ 2005;330:1115.

24. Cooper C, Eriksson JG, Forsen T, et al. Maternal height, childhood growth and risk of hip fracture in later life: a longitudinal study. Osteoporos Int 2001;12:623-9.

25. Roeder LM, Chow BF. Maternal undernutrition and its long-term effects on the offspring. Am J Clin Nutr 1972;25:812-21.

26. Hulshoff Pol HE, Hoek HW, Susser E, et al. Prenatal exposure to famine and brain morphology in schizophrenia. Am J Psychiatry 2000;157:1170-2.

27. Kuh D, Hardy R, Butterworth S, et al. Developmental origins of midlife grip strength: findings from a birth cohort study. J Gerontol $A$ Biol Sci Med Sci 2006;61:702-6.

28. Yliharsila $\mathrm{H}$, Kajantie $\mathrm{E}$, Osmond $\mathrm{C}$, et al. Birth size, adult body composition and muscle strength in later life. Int $J$ Obes (Lond) 2007;31:1392-9.

29. Sayer AA, Dennison EM, Syddall HE, et al. The developmenta origins of sarcopenia: using peripheral quantitative computed tomography to assess muscle size in older people. J Gerontol A Biol Sci Med Sci 2008;63:835-40.

30. Martin HJ, Syddall HE, Dennison EM, et al. Physical performance and physical activity in older people: are developmental influences important? Gerontology 2009;55:186-93.

31. Calado RT, Young NS. Telomere diseases. N Engl J Med 2009;361:2353-65.

32. Jennings BJ, Ozanne SE, Dorling MW, et al. Early growth determines longevity in male rats and may be related to telomere shortening in the kidney. FEBS Lett 1999;448:4-8.
33. Tarry-Adkins JL, Martin-Gronert MS, Chen JH, et al. Maternal diet influences DNA damage, aortic telomere length, oxidative stress, and antioxidant defense capacity in rats. FASEB $J$ 2008;22:2037-44.

34. Tarry-Adkins JL, Chen JH, Smith NS, et al. Poor maternal nutrition followed by accelerated postnatal growth leads to telomere shortening and increased markers of cell senescence in rat islets. FASEB J 2009;23:1521-8.

35. Finkel T, Holbrook NJ. Oxidants, oxidative stress and the biology of ageing. Nature 2000;408:239-47.

36. Chiavaroli V, Giannini C, D'Adamo E, et al. Insulin resistance and oxidative stress in children born small and large for gestational age. Pediatrics 2009;124:695-702.

37. Chung HY, Cesari M, Anton S, et al. Molecular inflammation: underpinnings of aging and age-related diseases. Ageing Res Rev 2009;8:18-30.

38. Tzoulaki I, Jarvelin MR, Hartikainen AL, et al. Size at birth, weight gain over the life course, and low-grade inflammation in young adulthood: northern Finland 1966 Birth Cohort study. Eur Heart $J$ 2008;29:1049-56.

39. Sattar N, McConnachie A, O'Reilly D, et al. Inverse association between birth weight and C-reactive protein concentrations in the MIDSPAN Family Study. Arterioscler Thromb Vasc Biol 2004;24:583-7

40. Canoy D, Pouta A, Ruokonen A, et al. Weight at birth and infancy in relation to adult leukocyte count: a population-based study of 5619 men and women followed from the fetal period to adulthood. J Clin Endocrinol Metab 2009;94:1916-22.

41. Chen JH, Martin-Gronert MS, Tarry-Adkins J, et al. Maternal protein restriction affects postnatal growth and the expression of key proteins involved in lifespan regulation in mice. PLoS ONE 2009;4:e4950.

42. Appel J, Potter E, Bhatia N, et al. Association of white matter hyperintensity measurements on brain MR imaging with cognitive status, medial temporal atrophy, and cardiovascular risk factors. Am J Neuroradiol 2009;30:1870-6.

43. Epel ES, Merkin SS, Cawthon R, et al. The rate of leukocyte telomere shortening predicts mortality from cardiovascular disease in elderly men. Aging (Albany NY) 2009;1:81-8.

44. Watters JL, Satia JA, da Costa KA, et al. Comparison of three oxidative stress biomarkers in a sample of healthy adults. Biomarkers 2009;14:587-95.

45. Barker DJP. Mothers, babies and health in later life. 2nd edn Edinburgh: Churchill Livingstone, 1998.

46. Bateson P, Barker D, Clutton-Brock T, et al. Developmental plasticity and human health. Nature 2004;430:419-21. 